\title{
Data extraction methods: an analysis of internal reporting discrepancies in single manuscripts and practical advice
}

\author{
Livia Puljak $^{\mathrm{a}, *}$, Nicoletta Riva ${ }^{\mathrm{b}}$, Elena Parmelli ${ }^{\mathrm{c}}$, Marien González-Lorenzo ${ }^{\mathrm{d}, \mathrm{e}}$, Lorenzo Moja ${ }^{\mathrm{f}}$, \\ Dawid Pieper ${ }^{g}$ \\ ${ }^{\text {a } C e n t e r ~ f o r ~ E v i d e n c e-B a s e d ~ M e d i c i n e ~ a n d ~ H e a l t h ~ C a r e, ~ C a t h o l i c ~ U n i v e r s i t y ~ o f ~ C r o a t i a, ~ Z a g r e b, ~ C r o a t i a ~}$ \\ ${ }^{\mathrm{b}}$ Department of Pathology, Faculty of Medicine and Surgery, University of Malta, Msida, Malta \\ ${ }^{\mathrm{c}}$ Department of Epidemiology, Lazio Regional Health Service - ASL Roma 1, Rome, Italy \\ ${ }^{\mathrm{d}}$ Department of Biomedical Sciences, Humanitas University, Milan, Italy \\ ' IBD Center, Humanitas Clinical and Research Center, Milan, Italy \\ ${ }^{\mathrm{f}}$ Policy, Access and Use (PAU), Essential Medicines and Health Products Department (EMP), World Health Organization, Geneva, Switzerland \\ ${ }^{\mathrm{g}}$ Witten/Herdecke University, School of Medicine, Cologne, Germany \\ Accepted 9 September 2019; Published online 18 September 2019
}

\begin{abstract}
Background: Data extraction from reports about experimental or observational studies is a crucial methodological step informing evidence syntheses, such as systematic reviews (SRs) and overviews of SRs. Reporting discrepancies were defined as pairs of statements that could not both be true. Authors of SRs and overviews of SRs can encounter reporting discrepancies among multiple sources when extracting data - a manuscript and a conference abstract, and a manuscript and a clinical trial registry. However, these discrepancies can also be found within a single manuscript published in a scientific journal.

Objectives: Hereby, we describe examples of internal reporting discrepancies that can be found in a single source, with the aim of raising awareness among authors of SRs and overviews of SRs about such potential methodological issues.

Conclusions: Authors of SRs and overviews of SRs should check whether the same information is reported in multiple places within a study and compare that information. Independent data extraction by two reviewers increases the chance of finding discrepancies, if they exist. We provide advice on how to deal with different types of discordances and how to report such discordances when conducting SRs and overviews of SRs. (c) 2019 Elsevier Inc. All rights reserved.
\end{abstract}

\section{Introduction}

Data extraction from reports about experimental or observational studies is a crucial methodological step informing evidence syntheses, such as systematic reviews (SRs) and overviews of SRs. Studies about data extraction for SRs have warned about possibility of extraction errors and high probability that two authors may extract different data [1,2]. The term "extraction errors" refers

Conflict of interest: The authors have no conflict of interest to declare.

* Corresponding author. Center for Evidence-based Medicine and Health Care, Catholic University of Croatia, Ilica 242, 10000 Zagreb, Croatia. Tel.: + 3850137066 00; fax: + 385013706601 .

E-mail addresses: livia.puljak@unicath.hr; livia.puljak@gmail.com (L. Puljak). both to subjective errors that are responsibility of reviewers (i.e., review authors extracted erroneous data) and objective errors within the manuscript that are responsibility of the primary study authors (i.e., different data reported in different sections within a manuscript). It has been reported that objective errors in primary studies are frequent $[3,4]$. These errors pose challenges to readers, reviewers, guideline developers, and can propagate into SRs.

A recently published SR about data extraction errors by Mathes et al. [5] indicated that extraction errors often have influence on the effect estimates. As a crucial element of systematic methodology, it has been recommended that two reviewers should conduct data extraction independently, or that one reviewer should do data extraction and another one verify the extraction [6,7]. The former should 


\section{What is new?}

\section{Key findings}

- Discrepancies can be found within a single research manuscript; these internal reporting discrepancies may negatively influence evidence synthesis.

- Internal reporting discrepancies may occur in various parts of the manuscript.

\section{What this adds to what was known?}

- It has been already indicated that research publications have errors, but implications of internal reporting discrepancies for evidence synthesis, such as systematic reviews and overviews of systematic reviews, have not been discussed before.

\section{What is the implication and what should change now?}

- Authors of evidence synthesis should be aware of the possibility of internal reporting discrepancies, check for multiple reports of the same information, contact authors to resolve such discrepancies, and transparently report them.

- Interventions for reducing internal reporting discrepancies would be welcome.

be the preferred method because Buscemi et al. [8] showed that the risk of extraction errors is significantly lower with two independent extractions compared to single extraction verified by another reviewer.

Discrepancies were defined as "pairs of statements that could not both be true" [9]. Authors of SRs and overviews of SRs can encounter reporting discrepancies among multiple sources when extracting data: between two manuscripts that describe the same study, and between a published manuscript and a conference abstract [10-12], and between trial registry entries and published manuscripts [13]. Attention to this has also been called in the new version (version 6) of the Cochrane Handbook for Reviews of Interventions, and its Chapter 5, where authors are warned that discrepancies may occur across multiple sources, and that authors should have a plan for resolving discrepancies [14].

However, as Cole et al. [15] have shown, discrepancies can also be found within a single manuscript published in a scientific journal. Even multiple peer reviews, copyediting, and revision of galley proof by original authors do not completely protect against the hazard of making one or more errors. The chance of finding discrepancies within a single manuscript is particularly high in complex SR, which often requires analysis of a large quantity of data from the same report/manuscript [16].

Cole et al. [15] have shown that it is difficult for readers of research manuscripts to notice discrepancies: in their study, they included 343 readers and found that $95.3 \%$ of discrepancies were missed. Reviewers of primary studies are, instead, a selected group of readers of primary articles: they carefully examine study data, extracting them in structured formats. This task facilitates spotting errors and discrepancies. If two reviewers are involved in data extraction, either independently or as verification, there is higher likelihood that errors and discrepancies will be found.

Hereby, we describe examples of objective errors and internal reporting discrepancies that SR and overviews of SRs authors can find within a single published manuscript, with the aim of raising awareness about such potential methodological issues. We also provide advice on how to deal with different types of discrepancies and how to report them when conducting SRs and overviews of SRs.

\section{Possible types of internal reporting discrepancies}

Internal reporting discrepancies occur when different data are reported in different sections within the same manuscript. In our experience, we identified 5 possible types of internal reporting discrepancies: abstract-text discrepancies, within-the-full-text discrepancies, text-figure discrepancies, text-table discrepancies, and discrepancies in multiple sections of the same manuscript. We reported in Table 1 some examples of each type of discrepancies.

Some of these discrepancies can be judged as trivial, such as differences encompassing second digit after the decimal place. However, other discrepancies, such as differences in study participants or number of included studies, can be relevant. They can have implication on key dimensions, such as generalizability of findings, or on key methods, such as the assumed degree of freedom to estimate heterogeneity.

\section{Take-home message: systematic review authors should be aware of potential internal reporting discrepancies}

Examples shown in this manuscript were discovered by a systematic approach because two independent data extractors found certain information in different places of the same manuscript. These represent an infinitesimal fraction of all published reviews and included errors within reviews. It is important that the meaning of systematic in "systematic reviews" is extended also to data checking, on top of search strategies and risk of bias assessment. If this approach to discrepancy becomes more frequent and consistent across review teams, discrepancies will not 
Table 1. Possible types of internal reporting discrepancies

\begin{tabular}{|c|c|c|}
\hline Type of discrepancies & Description & Examples \\
\hline $\begin{array}{l}\text { Abstract-text } \\
\text { discrepancies }\end{array}$ & $\begin{array}{l}\text { Discrepancies between the } \\
\text { abstract and the full-text } \\
\text { of a manuscript }\end{array}$ & $\begin{array}{l}\text { - In an MA [17], there was a discrepancy regarding the databases that were searched, } \\
\text { with the abstract being more detailed than the full text of the manuscript: } \\
\text { - The abstract reported "MEDLINE and the Cochrane Central Register of Controlled } \\
\text { Trials databases (1966 to March 2005)." } \\
\text { - In the text, it was stated "A comprehensive MEDLINE database search using Ovid } \\
\text { software (Ovid Technologies Inc, New York, NY) was performed to find human } \\
\text { studies published in the English language between } 1966 \text { and March } 2005 . " \\
\text { - In an MA [18], there was a minor numerical discrepancy regarding the Cl of the effect } \\
\text { size of aspirin in the prevention of stroke: } \\
\text { - In the abstract, it was stated that the risk ratio was " } 0.84 ; 95 \% \mathrm{Cl}, 0.64-1.11 . " \\
\text { - In the full text and Figure } 2, \text { it was reported that the risk ratio was "0.84, } 95 \% \mathrm{Cl} \\
0.63-1.11 \text { " } \\
\text { - In an MA [19], there was a discrepancy in the number of included patients: } \\
\text { - In the abstract, it was stated "six trials consisting of } 7,374 \text { patients with } \\
\text { diabetes." } \\
\text { - The sum of patients included in the six trials reported in Table } 2 \text { is } 7,907 . \\
\text { - In an MA [20], there was a discrepancy in the number of included RCTs: } \\
\text { - In the abstract, it was stated "nine RCTs with moderate to high methodological } \\
\text { quality contributed data to the analyses." } \\
\text { included in the MA. }\end{array}$ \\
\hline $\begin{array}{l}\text { Within-the-full-text } \\
\text { discrepancies }\end{array}$ & $\begin{array}{l}\text { Discrepancies in different } \\
\text { parts of the body of a } \\
\text { manuscript }\end{array}$ & $\begin{array}{l}\text { - In an RCT [21], there was a discrepancy in the number of patients who discontinued } \\
\text { treatment due to AEs: } \\
\text { - In the text ( } p \text { 75), it was stated "The reasons for discontinuation included the } \\
\text { following: AEs in } 13(10.3 \%) \text { and } 12(9.8 \%) \text { celecoxib- and diclofenac-treated } \\
\text { patients, respectively." } \\
\text { - In the text ( } p \text { 78), it was stated "Similar numbers of patients in each group dis- } \\
\text { continued the study medication because of treatment-related AEs (celecoxib, 17/ } \\
125[13.6 \%] \text {; diclofenac, } 16 / 123[13.0 \%]) . " \\
\text { - Furthermore, Figure } 1 \text { showed that the number of patients who discontinued } \\
\text { because of AEs was } 13 / 126 \text { in the celecoxib group (with a note that "One ran- } \\
\text { domized patient did not receive any study medication," therefore } 13 / 125 \text { ) and } 12 / \\
123 \text { in the diclofenac group. }\end{array}$ \\
\hline $\begin{array}{l}\text { Text-figure } \\
\text { discrepancies }\end{array}$ & $\begin{array}{l}\text { Discrepancies between a } \\
\text { figure and the full-text of } \\
\text { a manuscript }\end{array}$ & 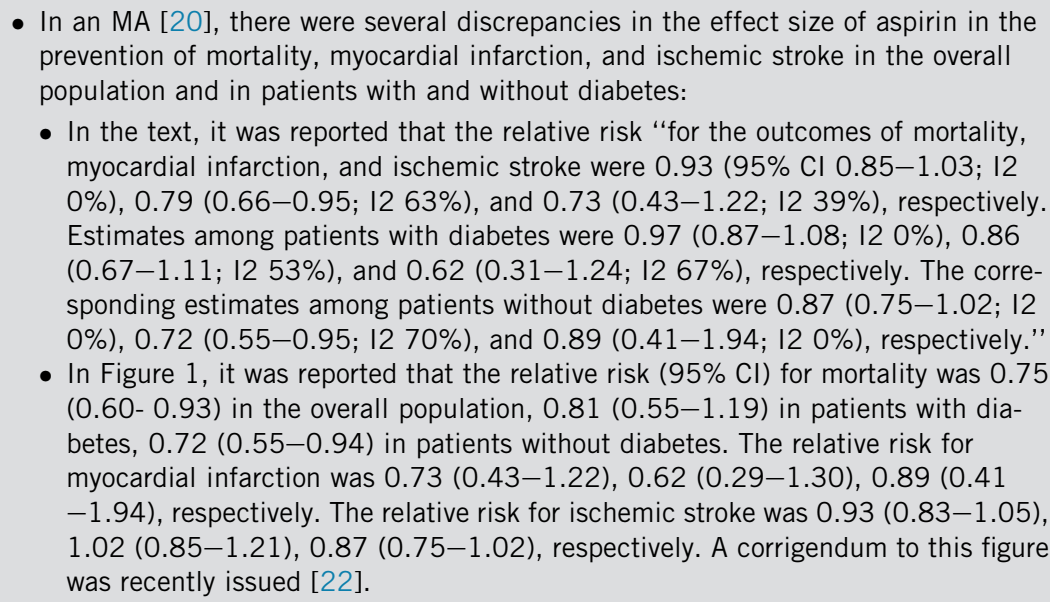 \\
\hline
\end{tabular}


Table 1. Continued

\begin{tabular}{|c|c|c|}
\hline Type of discrepancies & Description & Examples \\
\hline & & $\begin{array}{l}\text { - In an RCT [23], there was a discrepancy regarding the number of randomized } \\
\text { patients: } \\
\text { - In the text, it was stated "Of the } 22 \text { patients included in the RCT, four were } \\
\text { excluded for mobility assessments due to inability to understand instructions } \\
(n=2) \text { or due to missing data because measurements could not be performed due } \\
\text { to logistical reasons }(n=2 \text {; Figure } 1 \text { ). Hence, } 18 \text { patients were included in the } \\
\text { mobility assessments." } \\
\text { - The flow diagram (Figure } 1 \text { ) reported "Assessed for eligibility } N=22 \text { " and "ran- } \\
\text { domized } N=18 . \text {." The discrepancy in randomized patients encompasses a dif- } \\
\text { ference of almost } 20 \% \text { between the two figures ( } 18 \text { or } 22 \text { randomized patients). }\end{array}$ \\
\hline $\begin{array}{l}\text { Text-table } \\
\text { discrepancies }\end{array}$ & $\begin{array}{l}\text { Discrepancies between a } \\
\text { table and the full text of a } \\
\text { manuscript }\end{array}$ & $\begin{array}{l}\text { - In an MA [24], there was a discrepancy regarding the number of included } \\
\text { participants: } \\
\text { - In Table 1, the sum of participants was } 500,473 \text {. } \\
\text { - In the text, it was stated that "eight case-control studies with } 21,356 \text { cases and } \\
187,037 \text { controls and five cohort studies incorporating } 294,377 \text { participants were } \\
\text { included," which would sum up to } 502,770 \text { participants. Although this difference } \\
\text { in absolute terms is impressive (2,297 participants), in relative terms, the } \\
\text { difference is only } 0.5 \% \text {. }\end{array}$ \\
\hline $\begin{array}{l}\text { Multiple } \\
\text { discrepancies }\end{array}$ & $\begin{array}{l}\text { Discrepancies in multiple } \\
\text { sections of the same } \\
\text { manuscript }\end{array}$ & $\begin{array}{l}\text { - In an RCT [25], there were discrepancies regarding the number of included patients } \\
\text { overall and in the different arms: } \\
\text { - Abstract, Figure } 1 \text {, Table } 1 \text {, and Table } 5 \text { indicated that } 502 \text { patients were ran- } \\
\text { domized into four groups: dabigatran } 50 \mathrm{mg} \operatorname{BID}(n=105) \text {, dabigatran } 150 \mathrm{mg} \\
\text { BID ( } n=166) \text {, dabigatran } 300 \mathrm{mg} \text { BID ( } n=161) \text {, and warfarin }(n=70) \text {. } \\
\text { - Tables } 2 \text { and } 3 \text { indicated that the number of patients in the different groups was } \\
\text { dabigatran } 50 \mathrm{mg} \text { BID ( } n=107) \text {, dabigatran } 150 \mathrm{mg} \text { BID ( } n=169) \text {, dabigatran } \\
300 \mathrm{mg} \text { BID ( } n=169) \text {, and warfarin }(n=70) \text {, which would sum up to } 515 \\
\text { patients. }\end{array}$ \\
\hline
\end{tabular}

Abbreviations: $\mathrm{AE}$, adverse event, $\mathrm{BID}$, twice daily, $\mathrm{Cl}$, confidence interval, $\mathrm{MA}$, meta-analysis, $\mathrm{RCT}$, randomized controlled trial.

disappear, but at least they will not extend to secondary publications. It is highly likely that some of these discrepancies would not be found if the two reviewers looked for the information in the same place or if the reviewers did not check the entire manuscript. SR authors doing extraction should read the entire article because discrepancies may be found anywhere and not just in manuscript sections where data are typically found. Authors of SRs and overviews of SRs should also check whether data of interest are presented in multiple places in a manuscript of interest (abstract-text, different paragraphs of the text, text-figures/ tables) and contrast the same information reported in multiple sections.

\section{What should systematic review authors do in case of internal reporting discrepancies?}

Internal reporting discrepancies can have implications at the review level, which requires solutions to overcome challenges from the perspective of a reviewer.

Some of the discrepancies we encountered while conducting SRs and overviews of SRs were minor and unlikely to change the direction of results in an SR or overviews of
SRs, or the overall quality of evidence. However, some discrepancies were major, potentially reverting results in a meta-analysis or causing complete misinterpretation of results. It has also been previously emphasized that discrepancies can be a sign of deeply problematic studies, warranting further attention and verification [15]. The judgment of the relevance of discrepancies should be always collegial, involving more reviewers. The team should consider the interplay between the identified discrepancy and the results or other important dimensions of the review, such as risk of bias.

If authors extracting data from studies find discrepancies, they should attempt to contact corresponding authors of those studies to ask for clarification. If corresponding authors do not respond, the next step should be to try to contact all the authors of the study because this can increase the likelihood to obtain a response. It would be also valuable to copy the journal editor on this communication with author(s).

If the efforts to contact the study authors fail, authors of SR and overviews of SRs can choose to use several approaches, depending on the severity of the reporting discrepancy. If the reporting discrepancies are minor, the authors can include one set of data, with accompanying 
explanation about their choice. A second option is doing a sensitivity analysis to check whether inclusion of the other set of data could impact on the overall results, or a sensitivity without the involved data set. If the discrepancy has limited implications, we advise against an exclusion "without appeal" of the study, as this would be a disproportionate solution.

If the discrepancy is severe and choosing one data set over another would change direction of results, then it should be excluded from the main analysis and, eventually, included in a sensitivity analysis. For example, as in Cochrane reviews, a study can be classified as "awaiting classification" until the reporting discrepancy is resolved.

Irrespective of types of discrepancy, SR authors should always clearly describe what type of reporting discrepancy was found and what the implications were. In addition, authors of SRs and overviews of SRs should clearly report whom they tried to contact and how.

We summarized our suggestions for SR authors on how to address internal reporting discrepancies in Table 2.

\section{How to deal with internal reporting discrepancies in the literature?}

Internal reporting discrepancies can have also implications at the literature level, requiring solutions to signal, and potentially solve discrepancies.

Table 2. Suggestions for systematic review authors on how to address internal reporting discrepancies

SR authors should read the entire manuscript, check whether the same information is reported in multiple places within a manuscript, and compare that information

Two SR authors should conduct data extraction independently, to increase the likelihood of finding internal reporting discrepancies (if they exist)

If an internal reporting discrepancy is detected, a third SR author should be involved to confirm the presence of the discrepancy

The judgment of the relevance of internal reporting discrepancies should be always collegial, involving more SR authors

SR authors should attempt to contact the authors of studies with internal reporting discrepancies asking for clarification (eventually copying the journal editor in this communication)

If the internal reporting discrepancy is minor (e.g., unlikely to change the direction of results in an SR), SR authors can decide to include one of the two sets of data (with an accompanying explanation about their choice) and perform a sensitivity analysis (either including the other set of data or excluding the involved data set, to check the impact on the overall results)

If the internal reporting discrepancy is major (e.g., potentially reverting the results in a meta-analysis or causing complete misinterpretation of the results), the data set should be excluded from the main analysis (the study can be classified as "awaiting classification") and eventually included in a sensitivity analysis

SR authors should describe what type of internal reporting discrepancy was found, its implication, and their attempts to solve this discrepancy

Abbreviation: SR, systematic review.
Our experiences with spotting discrepancies and trying to solve them were that sometimes authors immediately indicated that there was indeed an error. Authors can be scrupulous, immediately notifying the journal the error and asking for a corrigendum. Other authors of manuscripts with discrepancies did not consider that a corrigendum was needed. Authors of SRs or overviews of SRs can also send a letter to the editor of the journal where the manuscript with discrepancies was found, indicating that there is a reporting discrepancy that should be clarified. It is in the interest of the research community to have access to publications without factual errors, and every effort to correct reporting discrepancies would be a valuable investment to increase the quality of the scientific literature.

Editors, peer reviewers, and manuscript authors have a crucial role in trying to prevent the appearance of internal reporting discrepancies. Peer reviewers, editorial staff and journal publishers should also check for potential discrepancy if certain information or data appear in multiple places in the manuscript. One potential solution is to create a checklist for copy editors and editorial staff, regarding multiple mentions of the same information and whether some discrepancies were spotted.

Some of the discrepancies we found, according to the contacted authors, were not the fault of authors, but happened after the acceptance of the manuscript. Corresponding authors are usually prompted by publishers to carefully review galley proofs to make sure that there are no errors [26]. This manuscript can also be a reminder to authors to carefully review the galley proofs before study publication, to check whether the same information is present in multiple places in the manuscript and whether there are any discrepancies that can still be corrected before final manuscript publication.

Journals may inadvertently contribute to discrepancies. Changes made by journal staff are not always clearly highlighted in the galley proof, making more difficult for the authors to spot potential errors. Some editors edit article page proofs without final approval by authors, which may lead to some discrepancies. The authors should always be given a chance to verify and approve editorial changes.

\section{Conclusion}

It has been already emphasized that most manuscripts published in the most important medical journals have errors [3]. The aim of this manuscript was not to simply repeat that errors can be found in a published manuscript but to raise awareness among authors of SRs and overview of SRs - and other types of studies which use data extraction from published reports about other studies - that discrepancies within a single manuscript can be more common than expected and that sometimes those discrepancies can have major consequences for conclusions of SRs and overviews of SRs. The importance of meticulous 
editorial and peer-review process, as well as galley proof checking, should also be highlighted as measures for preventing objective errors. As Cole et al. [15] have observed, "Even minor discrepancies should not be neglected, as they may be the tip of an error iceberg".

This manuscript included selected examples of discrepancies based on authors' experiences. By engagement of the wider research community, the next step could be to develop a taxonomy of discrepancies that SR and overview of SR authors may encounter. Such taxonomy could be used as a checklist or a decision tool for review authors and to help them to evaluate the nature and the impact of discrepancies.

\section{CRediT authorship contribution statement}

Livia Puljak: Conceptualization, Methodology, Writing - original draft, Writing - review \& editing. Nicoletta Riva: Conceptualization, Methodology, Writing - original draft, Writing - review \& editing. Elena Parmelli: Conceptualization, Methodology, Writing - original draft, Writing review \& editing. Marien González-Lorenzo: Conceptualization, Methodology, Writing - original draft, Writing - review \& editing. Lorenzo Moja: Conceptualization, Methodology, Writing - original draft, Writing - review \& editing. Dawid Pieper: Conceptualization, Methodology, Writing - original draft, Writing - review \& editing.

\section{Acknowledgments}

E.P., N.R., and L.M. were funded by the Italian Ministry (GR-2011-02348048 awarded to L.M. and E.P.). The funder had no role in study design, data collection and analysis, decision to publish, or preparation of the manuscript.

\section{References}

[1] Jones AP, Remmington T, Williamson PR, Ashby D, Smyth RL. High prevalence but low impact of data extraction and reporting errors were found in Cochrane systematic reviews. J Clin Epidemiol 2005;58:741-2.

[2] Carroll C, Scope A, Kaltenthaler E. A case study of binary outcome data extraction across three systematic reviews of hip arthroplasty: errors and differences of selection. BMC Res Notes 2013;6:539.

[3] Costantino G, Casazza G, Cernuschi G, Solbiati M, Birocchi S, Ceriani E, et al. Errors in medical literature: not a question of impact. Intern Emerg Med 2013;8:157-60.

[4] Altman DG. The scandal of poor medical research. BMJ 1994;308: 283-4.

[5] Mathes T, Klassen P, Pieper D. Frequency of data extraction errors and methods to increase data extraction quality: a methodological review. BMC Med Res Methodol 2017;17:152.

[6] Shea BJ, Grimshaw JM, Wells GA, Boers M, Andersson N, Hamel C, et al. Development of AMSTAR: a measurement tool to assess the methodological quality of systematic reviews. BMC Med Res Methodol 2007;7:10.

[7] AMSTAR checklist instructions. Available at https://amstar.ca/ Amstar_Checklist.php. Accessed October 19, 2019.

[8] Buscemi N, Hartling L, Vandermeer B, Tjosvold L, Klassen TP. Single data extraction generated more errors than double data extraction in systematic reviews. J Clin Epidemiol 2006;59:697-703.

[9] Francis DP, Mielewczik M, Zargaran D, Cole GD. Autologous bone marrow-derived stem cell therapy in heart disease: discrepancies and contradictions. Int J Cardiol 2013;168:3381-403.

[10] Saric L, Vucic K, Dragicevic K, Vrdoljak M, Jakus D, Vuka I, et al. Comparison of conference abstracts and full-text publications of randomized controlled trials presented at four consecutive World Congresses of Pain: reporting quality and agreement of results. Eur J Pain 2018;23:107-16.

[11] Saldanha IJ, Scherer RW, Rodriguez-Barraquer I, Jampel HD, Dickersin K. Dependability of results in conference abstracts of randomized controlled trials in ophthalmology and author financial conflicts of interest as a factor associated with full publication. Trials 2016;17:213.

[12] Dundar Y, Dodd S, Dickson R, Walley T, Haycox A, Williamson PR. Comparison of conference abstracts and presentations with full-text articles in the health technology assessments of rapidly evolving technologies. Health Technol Assess 2006;10. iii-iv, ix-145.

[13] Wandalkar P, Gandhe P, Pai A, Limaye M, Chauthankar S, Gogtay NJ, et al. A study comparing trial registry entries of randomized controlled trials with publications of their results in a high impact factor journal. J Am Med Assoc Perspect Clin Res 2017;8:167-71.

[14] Li T, Higgins JPT, Deeks JJ. Chapter 5. Collecting data. Cochrane Handbook for Systematic Reviews of Interventions. Version 6. The Cochrane Collaboration; 2019. Available at https://raining.cochr ane.org/handbook. Accessed October 19, 2019.

[15] Cole GD, Shun-Shin MJ, Nowbar AN, Buell KG, Al-Mayahi F, Zargaran D, et al. Difficulty in detecting discrepancies in a clinical trial report: 260-reader evaluation. Int J Epidemiol 2015;44:862-9.

[16] Mahtani KR, Jefferson T, Heneghan C. What makes a systematic review “complex"? Blog. BMJ EBM Spotlight. 2018. Available at https://blogs.bmj.com/bmjebmspotlight/2018/01/16/makes-systematicreview-complex/. Accessed October 19, 2019.

[17] Berger JS, Roncaglioni MC, Avanzini F, Pangrazzi I, Tognoni G, Brown DL. Aspirin for the primary prevention of cardiovascular events in Women and men: a sex-specific meta-analysis of randomized controlled trials. JAMA 2006;295:306-13.

[18] Butalia S, Leung AA, Ghali WA, Rabi DM. Aspirin effect on the incidence of major adverse cardiovascular events in patients with diabetes mellitus: a systematic review and meta-analysis. Cardiovasc Diabetol 2011;10:25.

[19] Younis N, Williams S, Ammori B, Soran H. Role of aspirin in the primary prevention of cardiovascular disease in diabetes mellitus: a meta-analysis. Expert Opin Pharmacother 2010;11:1459-66.

[20] Calvin AD, Aggarwal NR, Murad MH, Shi Q, Elamin MB, Geske JB, et al. Aspirin for the primary prevention of cardiovascular events: a systematic review and meta-analysis comparing patients with and without diabetes. Diabetes Care 2009;32:2300-6.

[21] Emery P, Koncz T, Pan S, Lowry S. Analgesic effectiveness of celecoxib and diclofenac in patients with osteoarthritis of the hip requiring joint replacement surgery: a 12-week, multicenter, randomized, double-blind, parallel-group, double-dummy, noninferiority study. Clin Ther 2008;30:70-83.

[22] Calvin AD, Aggarwal NR, Murad MH, Shi Q, Elamin MB, Geske JB, et al. Erratum. Aspirin for the primary prevention of cardiovascular events. A systematic review and meta-analysis comparing patients with and without diabetes. Diabetes Care 2009;32:2300-2306. Diabetes Care 2018;41:1321-2. 
[23] van den Elsen GA, Tobben L, Ahmed AI, Verkes RJ, Kramers C, Marijnissen RM, et al. Effects of tetrahydrocannabinol on balance and gait in patients with dementia: a randomised controlled crossover trial. J Psychopharmacol 2017;31:184-91.

[24] Zhu Y, Cheng Y, Luo RC, Li AM. Aspirin for the primary prevention of skin cancer: a meta-analysis. Oncol Lett 2015; 9:1073-80.
[25] Ezekowitz MD, Reilly PA, Nehmiz G, Simmers TA, Nagarakanti R, Parcham-Azad K, et al. Dabigatran with or without concomitant aspirin compared with warfarin alone in patients with nonvalvular atrial fibrillation (PETRO Study). Am J Cardiol 2007;100:1419-26.

[26] Matthews RJ, Bowen JM, Matthews RW. Successful scientific writing: a step-by-step guide for the biological and medical sciences. Cambridge, United Kingdom: Cambridge University Press; 2000. 